Mon. Not. R. Astron. Soc. 000, 16(2007) Printed 28 August $2018 \quad$ (MN LATEX style file v2.2)

\title{
WASP-1: A lithium- and metal-rich star with an oversized planet $^{\star}$
}

\author{
H. C. Stempels ${ }^{1} \dagger$ A. Collier Cameron ${ }^{1}$, L. Hebb ${ }^{1}$, B. Smalley ${ }^{2}$, and S. Frandsen ${ }^{3}$ \\ ${ }^{1}$ School of Physics 83 Astronomy, University of St Andrews, North Haugh, St Andrews, Fife, KY16 9SS, UK \\ ${ }^{2}$ Astrophysics Group, Keele University, Staffordshire, ST5 5BG, UK \\ ${ }^{3}$ Dept. of Physics and Astronomy, Arhus University, Denmark
}

Accepted XXXX. Received XXXX; in original form XXXX

\begin{abstract}
In this paper we present our results of a comprehensive spectroscopic analysis of WASP-1, the host star to the exoplanet WASP-1b. We derive $T_{\text {eff }}=6110 \pm 45 \mathrm{~K}$, $\log g=4.28 \pm 0.15$, and $[\mathrm{M} / \mathrm{H}]=0.23 \pm 0.08$, and also a high abundance of lithium, $\log n(\mathrm{Li})=2.91 \pm 0.05$. These parameters suggests an age for the system of $1-3 \mathrm{Gyr}$ and a stellar mass of $1.25-1.35 M_{\odot}$. This means that WASP-1 has properties very similar to those of HD 149026, the host star for the highest density planet yet detected. Moreover, their planets orbit at comparable distances and receive comparable irradiating fluxes from their host stars. However, despite the similarity of WASP-1 with HD 149026, their planets have strongly different densities. This suggests that gas-giant planet density is not a simple function of host-star metallicity or of radiation environment at ages of $\sim 2$ Gyr.
\end{abstract}

Key words: stars: planetary systems - stars: fundamental parameters - stars: individual: WASP-1

\section{INTRODUCTION}

Close-orbiting giant exoplanets that transit their parent stars offer unique insights into the interior structure and evolution of gas-giant planets. They are the only extrasolar planets whose radii and masses can be determined unambiguously from their light curves and radial-velocity variations. The discovery of the first such planet, HD 209458b (Charbonneau et al. 2000, Henry et al. 2000), established its gas-giant nature. Since then, 13 other transiting planets have been discovered, revealing a diverse range of densities and hence internal compositions. The Saturn-mass planet HD 149026b represents one extreme, having a high density requiring a substantial rock/ice core. At the other end of the density scale one finds HD 209458b, HAT-P-1b (Bakos et al. 2007) and WASP-1b (Collier Cameron et al. 2007). These planets have densities so low that even coreless models have difficulty reproducing their radii (Fortney et al. 2006, Burrows et al. 2006). Guillot et al. (2006) pointed out that the core masses inferred for then-known transiting planets show a weak correlation with the metallicity of the parent star. Burrows et al. (2006) find a similar trend. They also noted that WASP-1b, WASP-2b and XO-1b (McCullough et al. 2006) have very similar masses but substantially different

\footnotetext{
* Based on observations made with the Nordic Optical Telescope. † E-mail: Eric.Stempels@st-andrews.ac.uk
}

radii. In the absence of detailed spectroscopic abundance analyses for WASP-1 and WASP-2, however, it is difficult to determine a likely cause for their inflated radii.

WASP-1b has, in comparison with other planets, a very low density; it is 2.4 times as massive as HD $149026 \mathrm{~b}$, but also 3.3 times less dense. This poses a significant challenge to existing theoretical models. Burrows et al. (2006) find it hard to reconcile the planet's large radius $R_{p}=1.44 R_{\text {Jup }}$ (Charbonneau et al. 2007, Shporer et al. 2007) with evolutionary tracks even for coreless models with ten-timessolar atmospheric abundances and ages greater than 1.5 Gyr. This leads to a wide range of speculations. Could it be possible that the star (and by implication its planet) is relatively young, and thus still contracting? Could the strong irradiation of the planet inhibit contraction Guillot \& Showman 2002)? Can additional interior opacity due to enhanced atmospheric metallicity slow contraction (Burrows et al. 2006)? Or perhaps (as a counter-argument) can an increased interior molecular weight due to enhanced atmospheric metallicity accelerate contraction? Can tidal heating caused by orbital eccentricity (Bodenheimer, Lin \& Mardling 2001) or rotational obliquity (Winn \& Holman 2005) provide additional interior pressure support? Given so many theoretical candidate mechanisms for inflating a planet's radius, comparative studies of the rapidly-growing number of stars that host well-characterised transiting planets are essential if we are to identify the dominant environ- 


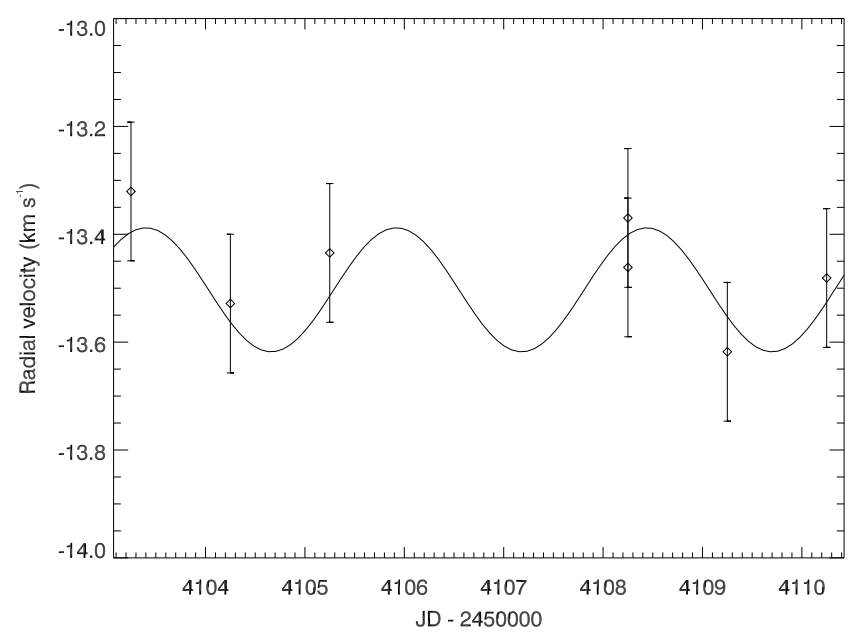

Figure 1. Radial velocity measurements from the seven obtained spectra of WASP-1, compared with the ephemeris determined by Collier Cameron et al. (2007).

mental and evolutionary processes that determine a mature gas-giant planet's internal structure and outer radius.

Here we present a detailed analysis of the spectrum of the parent star to WASP-1b, WASP-1 (= GSC 0226500107), with the goal of establishing its metallicity and evolutionary status. We use as our starting point the preliminary stellar parameters published by Collier Cameron et al. (2007). Although their analysis is roughly similar to the one presented here, the spectra they obtained from the SOPHIE spectrograph at OHP were compromised by background scattered-light contamination and uncertain continuum normalization, leaving the metallicity unconstrained. We also compare the metallicity, effective temperature and evolutionary age to those of the very similar host star of HD 149028b, and discuss whether the properties of the host stars give any useful clues to the strongly-contrasting densities of their close-orbiting gas-giant planets.

\section{OBSERVATIONS AND DATA REDUCTION}

WASP-1 was observed in early January 2007 at the $2.5 \mathrm{~m}$ Nordic Optical Telescope (NOT), as part of the science verification program of the newly commissioned spectrograph FIES (FIber Echelle Spectrograph). We obtained a total of seven spectra on six nights, with a spectral resolution of $R=47000$ and an exposure time of 20 minutes each, covering the wavelength region $4000-7350 \AA$ at a signal-to-noise of 40-50 in each spectrum.

The FIES spectrograph is a bench-mounted fiberfed echelle spectrograph, located in its own temperaturestabilized building and without any moving parts. With such a particular setup one obtains very stable spectra in a fixed format for which one of the authors (HCS) developed an automated data reduction system. This system uses PYTHON and PyRAF to access the echelle reduction routines of IRAF. The reduction is fine-tuned for the properties of FIES, and performs all necessary reduction steps, such as subtraction of biases and scattered light, flat-fielding, order extraction, normalization (including fringe-correction)
Table 1. Obtained parameters for WASP-1. See Sect. 3.3 for a description of how the uncertainties were derived.

\begin{tabular}{lcc}
\hline Parameter & Value & $\begin{array}{c}\text { Correlated } \\
\text { uncertainty }\end{array}$ \\
\hline$T_{\text {eff }}$ & $6110 \pm 45 \mathrm{~K}$ & $\pm 50 \mathrm{~K}$ \\
$\log g$ & $4.28 \pm 0.15$ & \pm 0.05 \\
{$[\mathrm{M} / \mathrm{H}]$} & $0.23 \pm 0.08$ & \pm 0.03 \\
$\log n(\mathrm{Li})$ & $2.91 \pm 0.05$ & \pm 0.05 \\
{$[\mathrm{Na} / \mathrm{H}]$} & $0.12 \pm 0.08$ & \pm 0.02 \\
{$[\mathrm{Si} / \mathrm{H}]$} & $0.26 \pm 0.06$ & \pm 0.01 \\
{$[\mathrm{Ti} / \mathrm{H}]$} & $0.30 \pm 0.16$ & \pm 0.01 \\
{$[\mathrm{Fe} / \mathrm{H}]$} & $0.26 \pm 0.03$ & \pm 0.02 \\
{$[\mathrm{Ni} / \mathrm{H}]$} & $0.24 \pm 0.07$ & \pm 0.02 \\
{$[\mathrm{Mg} / \mathrm{H}]$} & $0.10 \pm 0.05$ & \pm 0.04 \\
$v \sin i$ & $5.79^{*} \pm 0.35 \mathrm{~km} \mathrm{~s}^{-1}$ & - \\
$v_{\mathrm{rad}}$ & $-13.46 \pm 0.1 \mathrm{~km} \mathrm{~s}^{-1}$ & - \\
\hline
\end{tabular}

* Assuming a fixed value for the macroturbulence. Otherwise an upper limit (see Sect. 3.3 .

and wavelength calibration, leaving the observer with fullyreduced spectra ready for scientific analysis.

Using the associated wavelength calibration frames we corrected each spectrum for instrumental shifts. We also applied the correction for the heliocentric velocity and checked and calibrated the spectra against a radial velocity standard. For the purpose of determining the stellar parameters of WASP-1, we also created one high-quality spectrum with excellent signal-to-noise $(\sim 100)$ by combining the seven individual spectra into one.

\section{PROPERTIES OF WASP-1}

\subsection{Radial velocities}

The seven individual spectra we obtained provide an independent check of the sinusoidal radial-velocity variations detected in WASP-1 by Collier Cameron et al. (2007). In order to obtain the best accuracy, we cross-correlated high signalto-noise regions of the spectra on an order-by-order basis. In this analysis we excluded orders that contain atmospheric features. This allowed us to determine radial velocities with an accuracy of $\pm 125 \mathrm{~m} \mathrm{~s}^{-1}$. We find that the ephemeris determined by Collier Cameron et al. (2007) is compatible with our observations (see Fig. 11).

\subsection{Spectroscopic analysis}

Several detailed spectroscopic investigations of exoplanet hosts have been presented in the literature (among others, Gonzalez et al. 2001. Valenti \& Fischer 2005. Santos, Israelian, \& Mayor 2004, Santos et al.|2006). The primary difference between these studies is the choice of numerical methods and model atmospheres to determine which stellar parameters best describe the stellar spectrum. In this paper we choose to follow the methodology of one of these studies, namely Valenti \& Fischer (2005, hereafter VF05), which was also used in the studies of the transiting exoplanet systems XO-1 (McCullough et al. 2006) and HAT-P-1 (Bakos et al. 2007). We make identical assumptions and use of the same tools, techniques and grid of model atmosheres, allowing us 

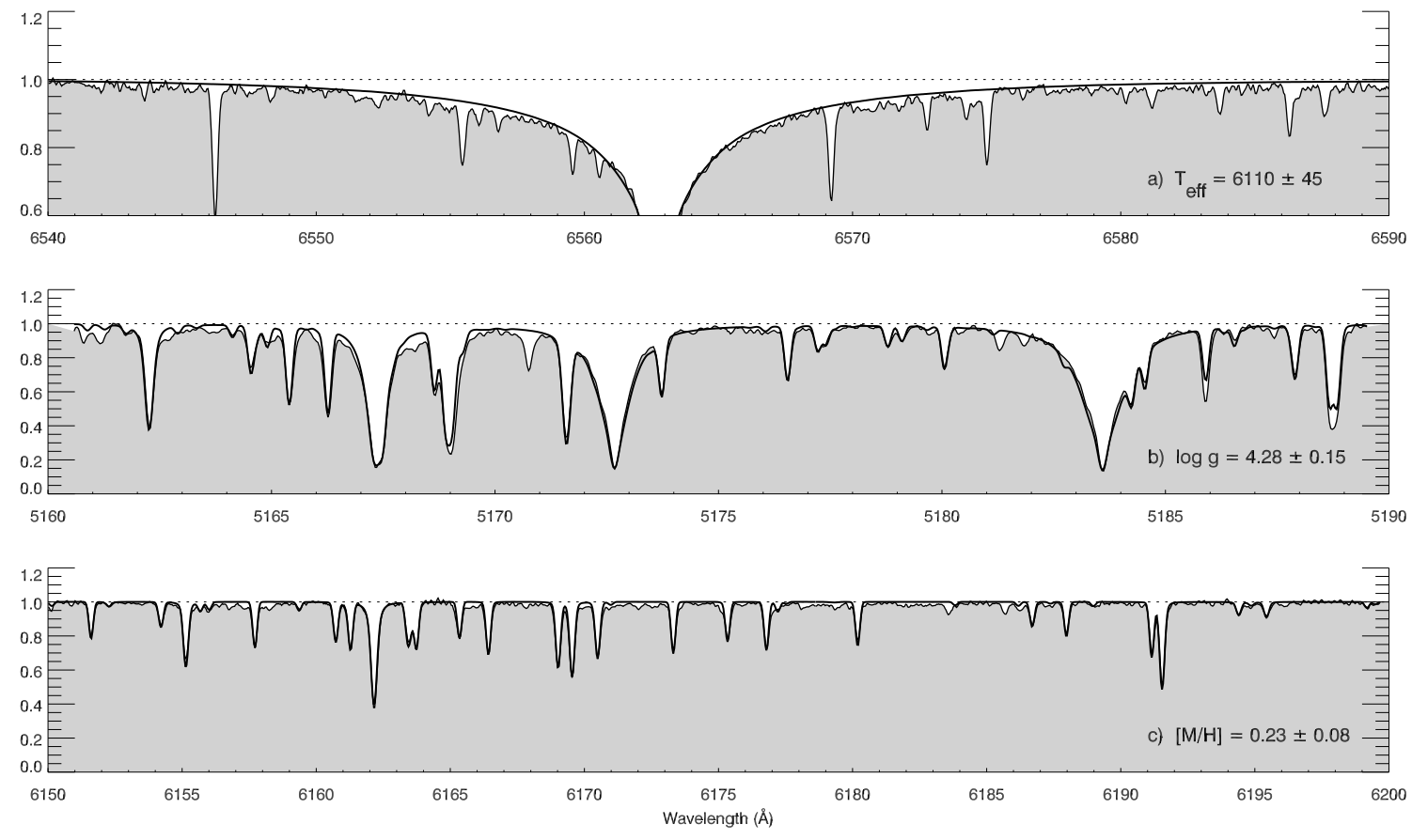

Figure 2. The above panels show a comparison of the observed spectrum (grey) with the synthetic spectrum based on the recovered parameters (solid, thick line). Panel a) shows the temperature-sensitive $\mathrm{H} \alpha 6563$ line, b) the $\mathrm{Mg}$ b 5175 triplet, sensitive to $\log g$ c) a section of the region containing a wealth of metal lines, sensitive to $[\mathrm{M} / \mathrm{H}]$ and d) the Li I 6708 line. Thes stellar parameters of WASP-1 were not determined from each panel individually, but from all spectral regions simultaneously (see text).

to obtain stellar parameters for WASP-1 that can be directly compared to the results of VF05.

We analysed our high-quality spectrum of WASP-1 with SME (Spectroscopy Made Easy, see Valenti \& Piskunov 1996), an IDL-based program that uses synthetic spectra and multi-dimensional least-squares minimization to determine the best set of stellar parameters (the effective temperature $T_{\text {eff }}$, the gravity $\log g$, the metallicity $[\mathrm{M} / \mathrm{H}]$, the projected radial velocity $v \sin i$, the systemic radial velocity $v_{\text {rad }}$, the microturbulence $v_{\text {mic }}$ and the macroturbulence $\left.v_{\text {mac }}\right)$ that describe an observed spectrum.

Successful calculation of synthetic spectra requires a grid of model atmospheres covering the parameter space of interest, as well as accurate line lists of atomic transitions. In analogy with VF05, we used for our analysis the routine for 3-dimensional interpolation on the Kurucz (1993) grid of LTE model atmospheres. Atomic line data was obtained from the VALD database (Piskunov et al. 1995; Kupka et al. 1999). To improve our ability to model the stellar spectrum in the spectral regions of interest (see below), we adjusted the oscillator strengths and broadening parameters for some of the lines in our line lists. This was done by comparing the NSO spectrum of the Sun (Kurucz et al. 1984) to a synthetic spectrum (using the parameters $T_{\text {eff }}=5770 \mathrm{~K}$, $\log g=4.44, v \sin i=1.4 \mathrm{~km} \mathrm{~s}^{-1}, v_{\mathrm{rad}}=0.4 \mathrm{~km} \mathrm{~s}^{-1}$ (gravitational blueshift), $v_{\text {mic }}=0.866 \mathrm{~km} \mathrm{~s}^{-1}$ and $v_{\mathrm{mac}}=3.57$ $\mathrm{km} \mathrm{s}^{-1}$ and solar abundances). We also checked our results against a high-quality spectrum of Procyon, obtained with FIES at the NOT (for stellar parameters, see Fuhrmann et al. 1997).

In order to use assumptions identical to those of VF05, we decoupled the correlation between microturbulence $v_{\text {mic }}$ and metallicity by fixing the value of $v_{\text {mic }}$ to $0.85 \mathrm{~km} \mathrm{~s}^{-1}$.
Similarly we followed their empirical relation for the value of the macroturbulence, giving $v_{\text {mac }}=4.5 \mathrm{~km} \mathrm{~s}^{-1}$ for a star with $T_{\text {eff }} \approx 6200 \mathrm{~K}$ (see also Sect. 3.3 for additional remarks on the choice of macroturbulence). We also released the elemental abundances of five elements ( $\mathrm{Na}, \mathrm{Si}, \mathrm{Ti}, \mathrm{Fe}$ and $\mathrm{Ni}$. As an initial guess we used the parameters determined by Collier Cameron et al. (2007), as well as $[\mathrm{M} / \mathrm{H}]$ $=0, v \sin i=0.5 \mathrm{~km} \mathrm{~s}^{-1}$, and $(\mathrm{Fe} / \mathrm{H})=-4.50$.

To constrain the full set of stellar parameters we identified three important wavelength regions (5160-5190 A, 6000-6200 $\AA$ and $6540-6590 \AA$ ). The first region contains the triplet of $\mathrm{Mg} b$ lines, primarily sensitive to $\log g$, while the second region contains a large number of well-isolated and unresolved spectral lines of a range of different elements, sensitive to $v_{\text {rad }}, v \sin i,[\mathrm{M} / \mathrm{H}]$, and the individual elemental abundances in particular.

The third region covers the broad $\mathrm{H} \alpha 6563$ line. Although this region was not included by VF05, we consider this region as a strong indicator to constrain effective temperature. The broadening of the outer wings of this line is sensitive to $T_{\text {eff }}$, and largely independent of other stellar parameters. Our synthetic synthesis calculations use the state-of-the-art hydrogen line broadening theory of Barklem, Piskunov, \& O'Mara (2000). Barklem et al. (2002) showed that this theory can accurately reproduce the Balmer line wings of stars with a range of different spectral types, and that it produces good agreement with the results of the infrared flux method (IRFM) developed by Blackwell, Petford, \& Shallis (1980), but with smaller error margins ${ }^{1}$ They also

1 While LTE simulations of Balmer line wings are widely used to determine stellar temperatures, Barklem (2007) recently pointed 
showed that there is a weak dependence on the model atmosphere used.

During our analysis we encountered difficulties in obtaining a good fit to the wings of the $\mathrm{Mg} b$ lines, sensitive to changes in $\log g$. While we obtained good agreement with other $\log g$-sensitive spectral features (notably the wings of Ca I 6122 and Ca I 6162), as well as with the spectra of the Sun and Procyon, the synthetic profiles for the $\mathrm{Mg} b$ lines remained too wide. Determining $\log g$ from the $\mathrm{Mg} \mathrm{b}$ lines alone yielded a very low value of $\log g=3.95$, which is strongly inconsistent with the photometrically determined radius of WASP-1. However, this apparent inconsistency can be reconciled by adjusting the elemental abundance of $[\mathrm{Mg} / \mathrm{H}]$.

Because our selected wavelength regions do not contain $\mathrm{Mg}$ lines other than $\mathrm{Mg} \mathrm{b}$, we determined the $\mathrm{Mg}$ abundance in WASP-1 from seven weak $\mathrm{Mg}$ lines in other regions of the spectrum ( $\mathrm{Mg}$ I $5528.4,5711.1,5785.3,5785.6$, $6318.7,6319.2$ and $6319.5 \AA$ ). We find $[\mathrm{Mg} / \mathrm{H}]=0.10$, indicating that $\mathrm{Mg}$ is indeed underabundant by 0.12 in WASP-1. Similar underabundances in $\mathrm{Mg}$ (and $\mathrm{Na}$ ) for metal rich stars and exoplanets hosts was reported by Gonzalez et al. (2001), although other comprehensive studies by Bensby et al. (2005) and Gilli et al. (2006) do not confirm such a trend. We then redetermined the stellar parameters of WASP-1, treating $[\mathrm{Mg} / \mathrm{H}]$ as a fixed parameter. This produced only minor changes in the derived stellar parameters, but also a good agreement between the observed and synthetic profiles around the $\mathrm{Mg}$ b line complex.

The final set of parameters we obtained from the analysis with SME are listed in Table 3.1 We also reproduce the synthetic spectra of the best choice of parameters in Fig 2 We would like to point out that all parameters were determined simultaneously across all three wavelength regions, thus obtaining a self-consistent solution.

\subsection{Error estimates and parameter correlations}

As VF05 point out, the formal uncertainties reported by SME strongly underestimate the true uncertainties because the value of the goodness-of-fit parameter $\chi^{2}$ is not dominated by the signal-to-noise of our spectra, but rather by uncertainties in the data reduction (for example, continuum fitting) as well as by inaccuracies in the synthetic spectra calculations. Thus, in analogy with VF05, we individually analysed our seven spectra of WASP-1 with SME, and quote the square root of the variance of the measurements of each parameter as the uncertainty in Table 3.1

Spectroscopically determined fundamental parameters tend to show strong correlations, and any uncertainty in one parameter may be propagated to other parameters. We investigated the magnitude of these correlations by varying $T_{\text {eff }}$ with $\pm 50 \mathrm{~K}$, and determining a new optimal solution at these temperatures, thus propagating the uncertainty in temperature to the other parameters. The correlated uncertainties (listed in Table 3.1) indicate that the uncertainties

out that it is unclear whether the assumption of LTE is applicable to the Balmer lines, and that non-LTE calculations might yield temperatures up to $100 \mathrm{~K}$ higher. due to parameter correlations are of similar order of magnitude as the uncertainties derived earlier from the scatter of measurements of the seven individual spectra.

In addition to propagating the uncertainties related to temperature, we also looked at the relation between $[\mathrm{Mg} / \mathrm{H}]$, $[\mathrm{M} / \mathrm{H}]$ and $\log g$, as these may be expected to correlate through the $\mathrm{Mg}$ b line complex. We find a clear correlation between $[\mathrm{Mg} / \mathrm{H}]$ and $[\mathrm{M} / \mathrm{H}]$, such that $[\mathrm{Mg} / \mathrm{H}]$ always shows a relative underabundance of about -0.1 with respect to $[\mathrm{M} / \mathrm{H}]$. On the other hand we see no indication that the value of $\log g$ correlates with $[\mathrm{Mg} / \mathrm{H}]$, mainly because each parameter is well constrained by diagnostics other than $\mathrm{Mg}$ b (i.e. the weak Mg lines and the broadening of Ca I 6122 and Ca I 6162).

One pair of parameters that shows a strong correlation are the assumed value of the macroturbulence $v_{\text {mac }}$ and the obtained projected rotational velocity $v \sin i$. This is no surprise, because the observed photospheric line profiles are a convolution of rotational and turbulent broadening. In our analysis we have assumed a value of $4.5 \mathrm{~km} \mathrm{~s}^{-1}$, similar to the choice of VF05. This value is in agreement with several other empirical relations for main-sequence type stars (see VF05). However, the parameters we derive for WASP1 could indicate that WASP-1 is starting to evolve off the main-sequence (see Sect. 4), which would imply a slight increase in $v_{\text {mac }}$, to approximately $5.5 \mathrm{~km} \mathrm{~s}^{-1}$. Using higher values for $v_{\text {mac }}$ would result in a lower value of the $v \sin i$, thus the value we quote in Table 3.1 should be considered an upper limit.

\subsection{Independent estimate of $T_{\text {eff }}$ from the IRFM}

In addition to the spectroscopic estimate, we also determined the effective temperature using the IRFM. This method allows for a nearly model-independent determination of $T_{\text {eff }}$ obtained from the integrated stellar flux at the Earth and a measurement at an infrared wavelength.

For WASP-1 we used the Tycho $B$ and $V$ magnitudes and the 2MASS magnitudes to estimate the integrated flux $\left(F_{\oplus}\right)$ by fitting Kurucz flux distributions. Since WASP-1 is relatively nearby and out of Galactic plane we assumed zero reddening. Integrating the fitted distributions gives a value of $F_{\text {tot }}=(6.2 \pm 0.5) \cdot 10^{-13} \mathrm{~W} \mathrm{~m}^{-2}$. Using the IRFM to determine the $T_{\text {eff }}$ for the $J, H$ and $K_{s}$ bands, we obtain an average $T_{\text {eff }}=6200 \pm 200 \mathrm{~K}$, which is in close agreement with the spectroscopic determination.

\section{EVOLUTIONARY STATUS}

The stellar parameters derived from the detailed spectroscopic analysis described above can be used to place WASP-1 in its evolutionary context, providing further constraints on the age of the host star and its planet. Both the effective temperature and the (photometrically determined) stellar radius are measured to high accuracy using well understood, robust physics that is largely model independent. Thus in Fig. 3, we plot a modified Hertzprung-Russel diagram in which the radius acts as a proxy for luminosity (as it is related to luminosity through the temperature) and compare the properties of WASP-1 to several different supersolar metallicity stellar evolution models (Girardi et al.|2002. 


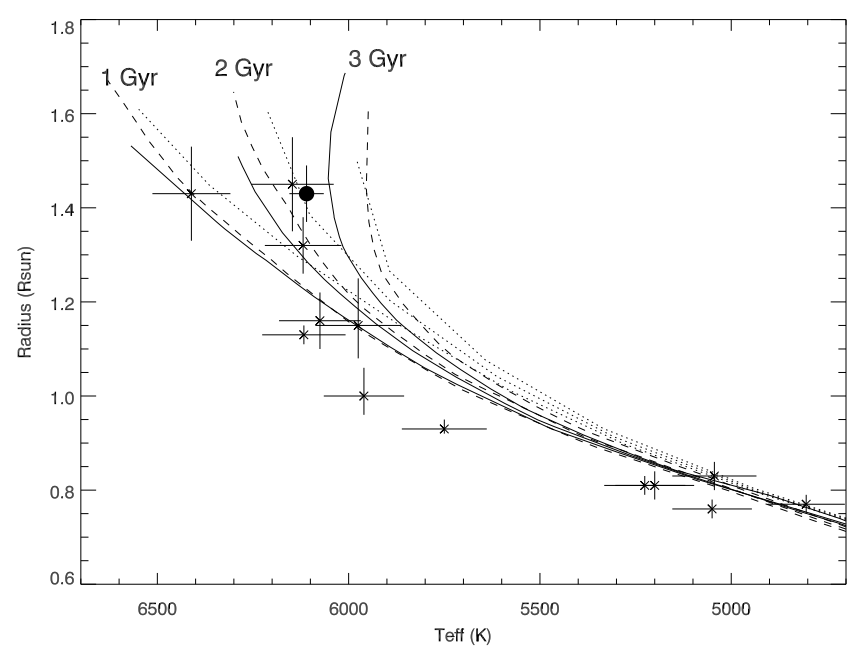

Figure 3. Radius versus $T_{\text {eff }}$ (a modified Hertzprung-Russel diagram) of the host stars of the 14 known transiting extra-solar planets Burrows et al. (asterisks, 2006), including WASP-1 (solid circle). Here we present isochrones for super-solar metallicity stellar evolution models from Girardi et al. (2002) (solid), Yi et al. (2001) (dotted), and Siess et al. (1997) (dashed). Given the uncertainties inherent in the models from various authors, the position of WASP-1 is consistent with an age of 1-3 Gyr.

Siess et al. 1997; Yi et al. 2001) between 1-3 Gyr. The position of WASP-1 in this diagram shows that its its age is likely to be in the range 1-3 Gyr and that its large stellar radius may be explained by the fact that the star is beginning to evolve off the main sequence.

We note that the mass initially adopted for WASP-1, $M=1.15 M_{\odot}$, (Collier Cameron et al. 2007) is marginally inconsistent with the $\log g$ found here, when combined with the latest estimates of the stellar radius $\left(R_{*}=1.453 \pm\right.$ $0.032 R$. Charbonneau et al. 2007, Shporer et al. 2007). A slightly higher mass of $M=1.25-1.35 M_{\odot}$ would allow all the measured values of $\log g$, temperature, and radius to be consistent given their errors.

\subsection{Lithium abundance}

An interesting feature in the spectrum of WASP-1 is the the strong lithium absorption line at $6708 \AA$. As stars only destroy lithium, the amount of photospheric lithium is often used as an age indicator. However, the accuracy of age estimates based on lithium abundances depends strongly on the efficiency with which stars deplete lithium. Stars such as WASP-1, with temperatures of $\sim 6100 \mathrm{~K}$, cannot deplete lithium very easily from their surface layers, because the bottom of the convection zone does not reach temperatures high enough to burn lithium. It is also cooler than $6300 \mathrm{~K}$, the lower limit of the so-called "Li dip" or "Boesgaard gap" (for example, Balachandran 1995) where lithium depletion is enhanced by an as yet unknown process. Still, early type stars do slowly deplete lithium during their main sequence lifetimes (Jones et al. 1999), allowing modest constraints to be placed on the age on WASP-1 based on the abundance of lithium present in the photosphere.

In the spectrum of WASP-1, Li I 6708 has an equivalenth width of $135 \mathrm{~mA}$. Using SME and the atmospheric parameters derived earlier, we find that this corresponds

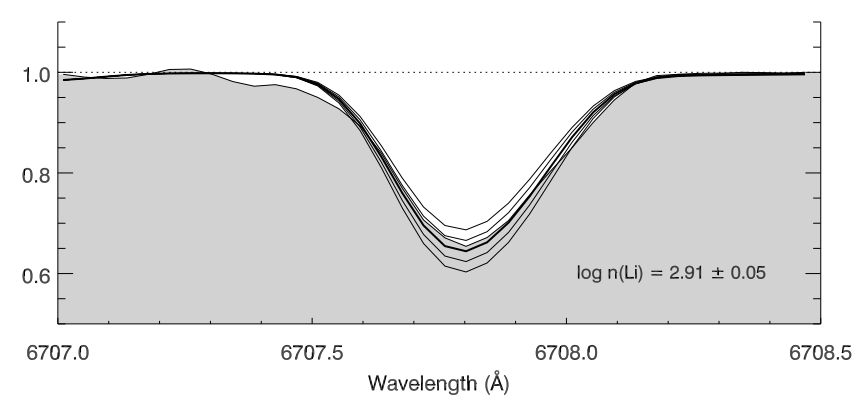

Figure 4. A range of synthetic line profiles for the Li I 6708 feature, in steps of 0.05 in $\log n(\mathrm{Li})$. The thick line corresponds to the optimum value, $\log n(\mathrm{Li})=2.91$. Changes of $\pm 50 \mathrm{~K}$ in the stellar effective temperature result in changes of \pm 0.05 in the lithium abundance (see text).

to an elemental abundance of $(\mathrm{Li} / \mathrm{H})=-9.09 \pm 0.05$, or $\log n(\mathrm{Li})=(\mathrm{Li} / \mathrm{H})+12=2.91 \pm 0.05$ in more conventional units (see Figure 4). We find a weak correlation between $T_{\text {eff }}$ and $\log n(\mathrm{Li})$, where a decrease in temperature by $50 \mathrm{~K}$ relates to a decrease in $\log n(\mathrm{Li})$ by 0.05 .

The value $\log n(\mathrm{Li})=2.91 \pm 0.05$ is less than the commonly adopted value of 3.2 for the primordial lithium abundance. Still, it is a the high end with respect to the study by Israelian et al. (2004), who compared the amount of lithium in exoplanet host stars with a set of reference stars. We also compared our value of $\log n(\mathrm{Li})$ with the empirical relations of lithium depletion in open clusters derived by Sestito \& Randich (2005). Their study shows that, for stars with temperatures of $6200 \pm 150 \mathrm{~K}$, lithium can be expected to reach $\log n(\mathrm{Li})=2.9$ after $1-3$ Gyr. This age estimate is consistent with what we derived earlier from evolutionary tracks.

\section{DISCUSSION}

Our spectroscopic analysis, together with the stellar mass and radius estimates inferred from previous studies, reveal a remarkable similarity between the physical parameters of WASP-1 and HD 149026 (see Table 5 The two stars appear to be very similar in both mass and evolutionary status. Sato et al. (2005) estimate the age of HD 149026 to be $2.0 \pm$ 0.8 Gyr. Also the environments in which the two planets orbit are remarkably similar. WASP-1b orbits slightly closer to a slightly cooler star, with the result that its blackbody effective temperature (assuming a low albedo and efficient redistribution and reradiation of heat from the night side) is just $80 \mathrm{~K}$ warmer than that of HD 149026b.

The structural differences between the two planets contrasts starkly with the similarities of the parent stars. The light, Saturn-mass HD 149026b requires a core mass of several tens of Earth masses to reproduce its small radius and high density (Sato et al. 2005, Fortney et al. 2006). WASP$1 \mathrm{~b}$ is more than twice as massive and has a much larger radius. This can only be reconciled with the models of Burrows et al. (2006) if it has little or no core, its atmosphere is metal-rich, and its age is less than $1.5 \mathrm{Gyr}$.

Although HD 149026 could be a factor 2 more metalrich than WASP-1 within the uncertainties, WASP-1 itself is substantially more metal-rich than the Sun. With this caveat in mind, we suggest that these two planets provide 
Table 2. A comparison between the stellar and planetary parameters of WASP-1 and HD 149026. Data for HD 149026 are taken from Charbonneau et al. (2006) and Sato et al. (2005). Data for WASP-1 are from Collier Cameron et al. (2007) and Charbonneau et al. (2007), except $T_{\text {eff, },},[\mathrm{Fe} / \mathrm{H}]$ and the age estimate, which are from this paper. The values of $T_{\mathrm{eff}, p}$ were determined assuming zero albedo and full redistribution of heat to the nightside.

\begin{tabular}{llcc}
\hline & & HD149026 & WASP-1 \\
\hline$M_{*}$ & $\left(M_{\odot}\right)$ & $1.3 \pm 0.1$ & $1.15_{-0.09}^{+0.24}$ \\
$R_{*}$ & $\left(R_{\odot}\right)$ & $1.45 \pm 0.1$ & $1.453 \pm 0.032$ \\
$T_{\text {eff }, *}$ & $(\mathrm{~K})$ & $6147 \pm 50 \mathrm{~K}$ & $6110 \pm 45 \mathrm{~K}$ \\
{$[\mathrm{Fe} / \mathrm{H}]$} & & $0.36 \pm 0.05$ & $0.26 \pm 0.03$ \\
Age & $(\mathrm{Gyr})$ & $2.0 \pm 0.8$ & $2.0 \pm 1.0$ \\
$M_{p}$ & $\left(M_{\mathrm{Jup}}\right)$ & $0.330 \pm 0.002$ & $0.79_{-0.06}^{+0.13}$ \\
$R_{p}$ & $\left(R_{\mathrm{Jup}}\right)$ & $0.726 \pm 0.064$ & $1.443 \pm 0.039$ \\
$\rho_{p}$ & $\left(\rho_{\mathrm{Jup}}\right)$ & 0.86 & 0.26 \\
orbital sep. & $(\mathrm{AU})$ & 0.042 & $0.0379 \pm 0.0042$ \\
$T_{\text {eff }, p}$ & $(\mathrm{~K})$ & 1740 & 1820 \\
\hline
\end{tabular}

a strong counter-example to the trend suggested tentatively by Guillot et al. (2006) and Burrows et al. (2006), in which core mass increases with metallicity of the host star. We conclude that the final balance between core and envelope mass in giant planets cannot be a simple function of stellar metallicity, nor of radiation environment. As Ikoma et al. (2006) conclude, the final composition of a planet is more likely to be dictated by the details of the disk environment in which the planet formed, and possibly the dynamical history following the envelope accretion phase, than by the composition of the parent star.

\section{ACKNOWLEDGMENTS}

We would like to thank the referees for constructive critisism that helped to improve this paper. HCS acknowledges support from the Swedish Research Council. The Nordic Optical Telescope is operated on the island of La Palma jointly by Denmark, Finland, Iceland, Norway, and Sweden, in the Spanish Observatorio del Roque de los Muchachos of the Instituto de Astrofisica de Canarias.

\section{REFERENCES}

Bakos G. A., et al., 2007, ApJ, 656, 552

Balachandran S., 1995, ApJ, 446, 203

Barklem P. S., 2007, A\&A, in press, arXiv:astroph/0702222

Barklem P. S., Piskunov N., O’Mara B. J., 2000, A\&A, 363, 1091

Barklem P. S., Stempels H. C., Allende Prieto C., Kochukhov O. P., Piskunov N., O'Mara B. J., 2002, A\&A, 385,951

Bensby T., Feltzing S., Lundström I., Ilyin I., 2005, A\&A, 433, 185

Blackwell D. E., Petford A. D., Shallis M. J., 1980, A\&A, 82, 249

Bodenheimer P., Lin D. N. C., Mardling R. A., 2001, ApJ, 548,466
Burrows A., Hubeny I., Budaj J., Hubbard W. B., 2006, ApJ, submitted, arXiv:astro-ph/0612703

Charbonneau D., Brown T. M., Latham D. W., Mayor M., 2000, ApJ, 529, L45

Charbonneau D., et al., 2006, ApJ, 636, 445

Charbonneau D., Winn J. N., Everett M. E., Latham D. W., Holman M. J., Esquerdo G. A., O'Donovan F. T., 2007, ApJ, 658, 1322

Collier Cameron A., et al., 2007, MNRAS, 375, 951

Fortney J. J., Saumon D., Marley M. S., Lodders K., Freedman R. S., 2006, ApJ, 642, 495

Fuhrmann K., Pfeiffer M., Frank C., Reetz J., Gehren T., 1997, A\&A, 323, 909

Girardi L., Bertelli G., Bressan A., Chiosi C., Groenewegen M. A. T., Marigo P., Salasnich B., Weiss A., 2002, A\&A, 391,195

Gilli G., Israelian G., Ecuvillon A., Santos N. C., Mayor M., 2006, A\&A, 449, 723

Gonzalez G., Laws C., Tyagi S., Reddy B. E., 2001, AJ, 121,432

Guillot T., Showman A. P., 2002, A\&A, 385, 156

Guillot T., Santos N. C., Pont F., Iro N., Melo C., Ribas I., 2006, A\&A, 453, L21

Henry G. W., Marcy G. W., Butler R. P., Vogt S. S., 2000, ApJ, 529, L41

Ikoma M., Guillot T., Genda H., Tanigawa T., Ida S., 2006, ApJ, 650, 1150

Israelian G., Santos N. C., Mayor M., Rebolo R., 2004, A\&A, 414, 601

Jones, B. F., Fischer, D., \& Soderblom, D. R. 1999, AJ, 117,330

Kupka F., Piskunov N., Ryabchikova T. A., Stempels H. C., Weiss W. W., 1999, A\&AS, 138, 119

Kurucz R., 1993, ATLAS9 Stellar Atmosphere Programs and $2 \mathrm{~km} / \mathrm{s}$ grid. (Kurucz CD-ROM No. 13) Cambridge, Mass.: Smithsonian Astrophysical Observatory

Kurucz R. L., Furenlid I., Brault J., Testerman L., 1984, NSO Atlas, Sunspot, New Mexico: National Solar Obs.

McCullough P. R., et al., 2006, ApJ, 648, 1228

Piskunov N. E., Kupka F., Ryabchikova T. A., Weiss W. W., Jeffery C. S., 1995, A\&AS, 112, 525

Santos N. C., Israelian G., Mayor M., 2004, A\&A, 415, 1153

Santos N. C., et al., 2006, A\&A, 458, 997

Sato B., et al., 2005, ApJ, 633, 465

Sestito P., Randich S., 2005, A\&A, 442, 615

Shporer A., Tamuz O., Zucker S., Mazeh T., 2007, MNRAS, in press, arXiv:astro-ph/0610556

Siess, L., Forestini, M., \& Dougados, C. 1997, A\&A, 324, 556

Valenti J. A., Fischer D. A., 2005, ApJS, 159, 141

Valenti J. A., Piskunov N., 1996, A\&AS, 118, 595

Winn J. N., Holman M. J., 2005, ApJ, 628, L159

Yi, S., Demarque, P., Kim, Y., Lee, Y., Ree, C. H., Lejeune, T., \& Barnes, S. 2001, ApJS, 136, 417

This paper has been typeset from a $\mathrm{T}_{\mathrm{E}} \mathrm{X} / \mathrm{IAT} \mathrm{T} \mathrm{X}$ file prepared by the author. 